\title{
Seguridad y control geopolítico: Crónica de la Iniciativa para la Prosperidad del Triángulo Norte de Centroamérica*
}

Security and geopolitical control: chronicle of the initiative for prosperity of the Northern Triangle of Central America

Fronteiras militarizadas: Crônica da iniciativa para a prosperidade do triangulo norte da América Central dos Afro-colombianos

\footnotetext{
* El autor es Doctor en Ciencias Sociales por la Universidad Autónoma Metropolitana (México). Investigador Titular del Centro de Estudios Superiores de México y Centroamérica, imparte docencia a nivel de posgrado. Sus líneas de investigación son: migraciones internacionales, integraciones México-Centroamérica; seguridad fronteriza y problemas del desarrollo. Es miembro del Sistema Nacional de Investigadores y de la Red Internacional de Migración y Desarrollo. Número de identificación ORCID: 00oo-00o2-5866-4534.

** Este artículo de investigación se inscribe en el marco del proyecto: «Flujos migratorios en las fronteras norte y sur de México y en tránsito hacia Estados Unidos: dinámicas de movilidad y políticas de contención», que inició en 2012 y concluyó en 2017, financiado por el Consejo Nacional de Ciencia y Tecnología (México), en la línea de Investigación Científica Básica, con el número de proyecto 0178078. Artículo de investigación recibido el 25.09.2017 y aceptado el 11.12.2017.
} 


\section{Cómo citar}

VILLAFUERTE SOLÍs, D. (2018). Seguridad y control geopolítico: Crónica de la Iniciativa para la Prosperidad del Triángulo Norte de Centroamérica. Revista CS. (24), 91-118.

DOI: http://dx.doi.org/10.18046/recs.i24.25o6. 
Resumen

Abstract

Resumo

El propósito de este artículo es presentar la concepción general del Plan Alianza para la Prosperidad del Triángulo Norte, uno de varios programas impulsados por el presidente Barack Obama para frenar la migración de niñas, niños y adolescentes centroamericanos que viajan sin acompañamiento de familiares, en el marco de una estrategia global de seguridad regional, que incluye la frontera sur de México como territorio de tránsito de migrantes y paso de drogas. También analiza el giro que se produce en la administración del presidente Donald Trump en materia migratoria cuyo énfasis es la seguridad y la militarización de las fronteras.

\section{PALABRAS CLAVE:}

Fronteras militarizadas; Migración de menores centroamericanos; Frontera sur de México; Alianza para la Prosperidad.

The purpose of this article is to present the general conception of the Plan Alliance for Prosperity of the Northern Triangle, one of several programs promoted by President Barack Obama to stop the migration of children and adolescents from Central America traveling unaccompanied by their family members, on the framework of a regional security global strategy, which includes the southern border of Mexico as a territory for the transit of migrants and drugs. The article also analyzes the changes in immigration matters that occurs with the arrival of President Donald Trump administration, which emphasis the security and militarization of borders.

\section{KEYWORDS:}

Military Frontiers; Migration of Central American Minors; Southern Border of Mexico; Alliance for Prosperity. 
O propósito deste artigo é apresentar a concessão geral do Plano Aliança para a prosperidade do Triangulo Norte, um dos vários programas impulsados pelo presidente Barak Obama para frear a migração de meninos, meninas e adolescentes centro americanos que viajam sem acompanhamento de familiares, no marco de uma estratégia global de segurança regional, que inclui a fronteira sul do México como território de transito de migrantes e passagem de drogas. Também analisa o giro que se produz na administração do presidente Donald Trump na matéria migratória cuja ênfase é a segurança e a militarização das fronteiras.

\section{PALAVRAS CHAVES:}

Fronteiras militarizadas; Migração de menores centro americanos; Fronteira sul do México; Aliança para a prosperidade. 


\section{Introducción}

En el verano de 2014, con la crisis de los menores migrantes centroamericanos aprehendidos en la frontera sur de Estados Unidos, se abrió una nueva etapa en la estrategia intervencionista de Estados Unidos. A los gobiernos de Guatemala, El Salvador y Honduras se les exige dar respuestas a la migración de niñas, niños y adolescentes que viajan de forma irregular y sin acompañamiento de estos países. Estos gobiernos no tienen la capacidad de ofrecer una solución, porque no se trata de un problema coyuntural sino de un asunto de carácter estructural de hondas raíces incubadas durante muchos años, que al hacerse masivo y endurecerse las medidas de control en la frontera sur de Estados Unidos hace crisis.

La respuesta de los gobiernos del llamado Triángulo Norte de Centroamérica (Guatemala, El Salvador y Honduras) fue la creación del Plan Alianza para la Prosperidad del Triángulo Norte-APTN-. Este plan combinado con acciones de contención-detención en la frontera sur de México y a lo largo de las rutas migratorias del país, fue oficialmente nominado Programa Frontera Sur; algunos lo llaman Plan Sur.

La elaboración y aprobación del APTN contó con la asesoría del gobierno de Estados Unidos y del Banco Interamericano de Desarrollo -BID-. Para tal efecto, se instrumentó un despliegue de actividades técnicas, políticas y diplomáticas de funcionarios del más alto nivel y al fin la APTN fue presentada y aprobada en Washington en diciembre de 2014. Luego vinieron los ajustes por cada uno de los países, sobre una programación más detallada de acciones en lugares específicos. En el transcurso de 2015 el gobierno de Barack Obama gestionó ante el congreso de su país un apoyo por mil millones de dólares para los tres países involucrados. Pasó año y medio y en diciembre de 2016 el Congreso de Estados Unidos aprobó un monto de 750 millones de dólares con una serie de condicionantes, sobre todo de combate a la corrupción.

El objetivo central de este artículo es reflexionar sobre las políticas de contención migratoria del gobierno de Estados Unidos. En particular la implementación de la APTN, que forma parte del marco general de la política de seguridad. Una política de seguridad que se aplica con especial énfasis en las fronteras norte y sur de México pero que se extiende a todo el territorio mexicano y centroamericano. También es objeto de atención el giro que se produce en el gobierno de Donald Trump, que da continuidad a las medidas de seguridad del presidente Obama, pero con marcado énfasis en la militarización. 


\section{El principio}

En el principio todo fue emergencia, alarma y preocupación del gobierno de Barack Obama por una oleada de migrantes menores de edad no acompañados de familiares. Las detenciones en la frontera sur de Estados Unidos fueron masivas, más de 60 mil, al grado que superaron la capacidad de los centros de detención y tuvieron que habilitar espacios de la Guardia Nacional y se contrataron abogados para avanzar más rápido en la dictaminación de casos y proceder a la deportación. Este fenómeno provocó la búsqueda de responsables y todo apuntó a los gobiernos de Guatemala, El Salvador y Honduras, también a México por dejar pasar. Se insistió mucho en que la causa fundamental era la violencia, por ello buena parte de las acciones en el marco de la APTN van dirigidas a este tema.

En el fondo se trataba de justificar y derivar más recursos a la Iniciativa de Seguridad Regional para Centroamérica-CARSI, por sus siglas en inglés-, que venía operando desde 2008. Sin embargo, se quería presentar la cara del desarrollo económico y la prosperidad. La hipótesis es que la APTN consistía en proponer una especie de ASPAN -Acuerdo para Seguridad y Prosperidad de América del Norte-para los tres países centroamericanos con la participación de México y Estados Unidos.

Acicateados por la emergencia migratoria de menores de edad, el 25 de julio de 2014 los presidentes de Guatemala, El Salvador y Honduras se reunieron con Barack Obama para encontrar una salida a este fenómeno. Los meses que siguieron fueron de trabajo coordinado entre varias instancias de los gobiernos involucrados y el Banco Interamericano de Desarrollo-BID-, el resultado fue la presentación formal de la APTN en el mes de noviembre en Washington.

El 26 de febrero de 2015, en Tela, Honduras, ocurre el primer encuentro de presidentes de Honduras, Guatemala, y el vicepresidente de El Salvador, para presentar el primer borrador de la iniciativa. En esta cumbre, además de los presidentes centroamericanos, se contó con la asistencia de la representación de México, Panamá y República Dominicana, del Banco Interamericano de Desarrollo y del representante especial para asuntos comerciales y empresariales del Departamento de Estado de Estados Unidos, entre otros.

Con optimismo desbordante, el presidente de Guatemala, Otto Pérez Molina, expresó: «hoy es un día histórico para la región, porque dos grandes temas son los que nos unen, el primero es el Encuentro de la Alianza para la Prosperidad; el otro es que estamos dando el paso que hemos esperado por 64 años en Centroamérica con la unión aduanera entre Honduras y Guatemala» («Hoy es un día histórico...»).

Camilo Atala, banquero hondureño y presidente del Consejo Empresarial de América Latina, capítulo Honduras, refirió que «con esta iniciativa se busca pro- 
mover la inversión, la integración económica, el desarrollo del capital humano, el fortalecimiento de las instituciones y la seguridad de los pueblos» («Camilo Atala: Empresarios...»).

En los primeros días de marzo de 2015, Joe Biden, vicepresidente de Estados Unidos, viajó a Guatemala para reunirse con sus homólogos de Guatemala, El Salvador y Honduras, junto con Luis Alberto Moreno, presidente del BID. La idea básica de la reunión era conocer con detalle las acciones de cada eje del plan, que son:1) dinamizar el sector productivo, 2) generar empleos, 3) mejorar la seguridad, y 4) fortalecer las instituciones del Estado. ${ }^{1}$ Con la información se presentarían argumentos ante el Congreso de Estados Unidos para la aprobación de los mil millones de dólares solicitados por el presidente Obama.

En efecto, el 3 de marzo el documento fue aprobado por los equipos de los tres países en medio de la visita de dos días a Guatemala del vicepresidente Joe Biden. El presidente guatemalteco, Pérez Molina, refirió que «el 16, 17 y 18 de marzo se van a dar detalles del plan en Washington con los equipos de cada país para llegar al detalle de las acciones que se van hacer y el costo de las mismas» («Cancilleres llevan borrador...»). El día 15 de marzo los cancilleres de los tres países se reunieron en Washington con el propósito de presentar al vicepresidente Biden las acciones del primer borrador de la iniciativa con las acciones específicas a realizar por cada país que suponen evitarían la migración.

En medio de la crisis migratoria y del cabildeo para el financiamiento de la APTN, a principios de abril de 2015 se anunció la llegada de marines estadounidenses a Honduras con el propósito de reforzar la lucha antidrogas. La poca trasparencia con la que el gobierno manejó el asunto dio lugar a especulaciones sobre la autorización del gobierno hondureño, y si realmente había habido una petición formal del gobierno de Estados Unidos. Poco a poco se fue filtrando información a los medios; por ejemplo, una nota que trascendió fue que Estados Unidos estaba estudiando establecer en Honduras un contingente de Fuerzas Especiales para Latinoamérica: «la unidad se denominaría Fuerza de Tarea Sur de Marines con Propósito Especial Aire-Tierra y tendría como objetivo responder a crisis en la región» («Honduras estudia ser...»). El vocero de las fuerzas armadas de Honduras, coronel José Antonio Sánchez, confirmó la propuesta de Estados Unidos. Esta nota fue complementada con la noticia del arribo de marines de Estados Unidos a Honduras, en el marco de una negociación con el general John Kelly del Comando Sur, los integrantes de esta 
fuerza de tarea serían elementos de la Fuerza Expedicionaria Marines Número II, con sede en Carolina del Norte (Rodríguez, 7 de abril de 2015).

Días después, el 14 de abril se conoció que el gobierno de Honduras había aceptado la presencia de las fuerzas armadas estadounidenses en la Mosquitia y Trujillo. El presidente Juan Orlando Hernández declaró que «estamos ya terminando la planificación y es obligación de ellos (los estadounidenses), como le he dicho al presidente (Barack) Obama, y al vicepresidente Joe (Biden), ayudarnos en las escuelas y en la prevención de todos los problemas que deja el narcotráfico en el país» («Marines de Estados Unidos...).

Los problemas de corrupción en los países de la región, que en Guatemala propició la renuncia del presidente Otto Pérez Molina, así como la discusión en el Congreso de Estados Unidos han retrasado la aprobación y entrega de recursos para la APTN. En este marco, en 2015 el Congreso de Estados Unidos aprobó un paquete de ayuda a la región por 750 millones de dólares, esta ayuda comenzó a llegar a principio de 2017. Mientras tanto, en diciembre de 2016 se decretó una resolución que mantuvo los fondos al nivel de 2016 hasta abril de 2017, y en mayo de este año se aprobaron otros 655 millones de dólares para el resto del año fiscal, que concluyó el 30 se septiembre. Este último recurso representa una disminución con respecto a la asignación de 2016 (Beltrán, 2017) («Monitoreando Programas...»). Por su parte, los países del área han asignado 5.300 millones en dos años (2015 y 2016), monto que incluye un préstamo del BID por 850 millones de dólares.

En el Documento de Trabajo del Plan de la Alianza para la Prosperidad del Triángulo Norte, de septiembre de 2015, queda clara la carga financiera que significa para los tres países centroamericanos:

El Plan representa un compromiso para el periodo 2016-2020 por más de US\$22,000 millones que incluyen actividades, acciones y proyectos en las cuatro líneas estratégicas. El Plan ha sido concebido bajo la premisa de que este representa el esfuerzo conjunto de los gobiernos del Triángulo Norte, el sector privado nacional e internacional, los organismos multilaterales y otros socios de desarrollo en la región. Asimismo, hemos planteado desde los orígenes del Plan que la mayor parte de la financiación de este Plan vendrá de nuestros propios recursos (Plan de la Alianza para la Prosperidad del Triángulo Norte, 2015: 10).

Como se sabe, los países del Triángulo norte presentan serias dificultades para financiar proyectos, y normalmente recurren a la cooperación internacional. Sin embargo, aunque en este caso el gobierno de Estados Unidos se ha comprometido a otorgar fondos, siempre estarán en función de sus intereses geopolíticos. Como se puede observar en el cuadro 1, la asistencia de Estados Unidos, a pesar de que se 
duplicó entre 2014 y 2016, resulta a todas luces insuficiente para hacer frente a un fenómeno de enorme complejidad como la migración. El cuadro también muestra un incremento importante del presupuesto en materia de seguridad y asistencia militar, del orden de $116 \%$, lo que imprime un sello particular a la APTN.

\begin{tabular}{|c|c|c|c|}
\hline \begin{tabular}{l|l} 
CUADRO 1 & Asistencia pa
\end{tabular} & troamérica 2014 & o16 (dólares) & \\
\hline Concepto & Aprobado 2014 & Solicitado 2016 & Aprobado 2016 \\
\hline Ayuda militar o seguridad & 16552000 & 26850000 & 30090000 \\
\hline Ayuda militar y seguridad & 100000000 & 205000000 & 222000000 \\
\hline $\begin{array}{l}\text { Asistencia económica o } \\
\text { fortalecimiento institucional }\end{array}$ & 224843000 & 769522000 & 497910 ooo \\
\hline Total & 341407014 & 1001732000 & 750000000 \\
\hline
\end{tabular}

Fuente: Beltrán, 2015.

Desde el 2015, cuando se aprueba la APTN, la realidad no ha cambiado sustancialmente. La migración continúa y los menores de edad siguen su peregrinar, aun a costa de perder la vida; aunque ciertamente el número de detenciones, tanto en México como en la frontera sur de Estados Unidos, disminuyó notablemente en 2017 en comparación con el año precedente, como se puede apreciar en el cuadro 2. El nuevo énfasis de la APTN, centrado en la seguridad nacional y la militarización de las fronteras, no detendrá la migración; por el contrario, se requiere de un proyecto de desarrollo incluyente, orientado fundamentalmente a mujeres y hombres jóvenes.

\section{El giro de la APTN con el presidente Trump: ¿hacia una Plan Columbia para la región?}

La primera señal de cambio fue en febrero de 2017: a partir de la visita a México de Rex Tillerson, Secretario de Estado, y John Kelly, Secretario de Seguridad, quienes se reunieron primero con sus contrapartes mexicanas: Luis Videgaray, de Relaciones Exteriores, y Miguel Ángel Osorio Chong, secretario de Gobernación. Luego serían recibidos en Los Pinos por el presidente Peña Nieto. 
El jueves 15 de junio de 2017, a pocos meses de haber asumido Donald Trump la presidencia de Estados Unidos, se llevó a cabo la Conferencia de Prosperidad y Seguridad en Miami, Florida. El ingrediente nuevo es que a la prosperidad se añade el tema de seguridad, ya explícito en la ayuda de los 750 millones de dólares otorgada por el gobierno de Estados Unidos para la APTN. De esta manera comienza a dibujarse una especie de Acuerdo para la Seguridad y Prosperidad para el Triángulo Norte de Centroamérica -ASPTNCA-, donde de manera más clara se coloca el componente de seguridad.

Esta conferencia fue convocada de manera conjunta por Estados Unidos y México. Entre los funcionarios de Estados Unidos de mayor nivel estuvieron el vicepresidente Mike Pence, el secretario de Estado Rex Tillerson, y el secretario de seguridad John Kelly. Por México participaron los secretarios de gobernación, Miguel Ángel Osorio Chong, de Relaciones Exteriores, Luis Videgaray, quien habló del tema de integración energética, y de Hacienda, José Antonio Meade. La presencia de estos funcionarios viene a corroborar el compromiso que el gobierno de México ha venido asumiendo con el de Estados Unidos en materia de migración y seguridad en la región Triángulo Norte-frontera sur de México, pero ahora sorprende la actitud colaboracionista en un ambiente hostil donde el gobierno de Trump ha decidido construir el muro fronterizo y renegociar a su favor el Tratado de Libre Comercio de América del Norte - TLCAN.

Previo a la reunión, Miami Herald publicó un documento elaborado por Rex Tillerson, John Kelly y Luis Alberto Moreno, presidente del BID, una pieza fundamental en el financiamiento de la APTN. Este documento proporciona claves importantes para entender por dónde se encaminan los esfuerzos del gobierno de Estados Unidos: convertir la APTN en una especie de Plan Colombia para estos países. Una parte del texto es claro en este sentido:

Contrariamente a lo que muchos predijeron al inicio, el Plan Colombia funcionó, a medida que tres consecutivas administraciones colombianas mostraron una fuerte determinación política, y pusieron en vigor un programa sostenido para desmantelar los cárteles, aumentar la seguridad, y promover la actividad económica.

Creemos que una estrategia similar puede funcionar en Centroamérica. Durante estos últimos dos años, el Banco Interamericano de Desarrollo (BID) ha estado apoyando la Alianza para la Prosperidad en el Triángulo Norte. Esencialmente, este plan busca ayudar a El Salvador, Guatemala y Honduras a alcanzar lo que Colombia logró: volver a tomar control de su territorio, poner fin al ciclo de violencia, corrupción e impunidad, ganar la confianza de los inversionistas y crear condiciones para un crecimiento sostenido e inclusivo (Tillerson, Kelly y Moreno, 2017). 
El sello del Plan Colombia ${ }^{2}$ ha sido la presencia de asesores y efectivos militares de Estados Unidos, además del aprovisionamiento de equipo, armas y entrenamiento militar. De manera que plantear una estrategia de esta naturaleza para Centroamérica significa la intervención directa de tropas de Estados Unidos en la región, y posiblemente en México.

En concordancia con lo anterior, un día antes de iniciar la Conferencia de Miami, WOLA difundió un documento elaborado por Beltrán (2017a), en el que expresa cuatro preocupaciones fundamentales sobre la cumbre de Centroamérica. La primera se refiere a la reiteración de la continuidad del compromiso del gobierno de Estados Unidos con los países del Triángulo Norte, aunque contradictoriamente el apoyo financiero se reduce: 750 millones de dólares para el año fiscal 2016; 655 millones para 2017; y la propuesta para 2018 es de 468 millones de dólares. Aunado a esta reducción en la asistencia, se está considerando no renovar el acuerdo de estatus temporal para migrantes salvadoreños y hondureños (Temporary Protected Status, TPS) que se han acogido a este acuerdo, que suman alrededor de 300.000 personas.

La segunda preocupación es la posibilidad de un giro en la cooperación de Estados Unidos «hacia un enfoque más militarizado y centrado en la seguridad». Esta situación, dice Beltrán (2017a), «podría resultar en un incremento del papel del Comando Sur en Centroamérica. Esto es particularmente preocupante dada la retórica del secretario de seguridad interna Kelly..., quien en varias ocasiones ha caracterizado las causas de la violencia en Centroamérica como producto del narcotráfico».

En esta línea de reflexión, la tercera preocupación se refiere, según Beltrán (2017a), a «el rol sobredimensionado del Departo de Seguridad Interna en discusiones y decisiones de política exterior que deben estar bajo el liderazgo del Departamento de Estado». Finalmente, la cuarta preocupación tiene que ver con «el papel de México como co-anfitrión de la conferencia genera preocupaciones sobre la creciente participación del país en el control de la migración desde Centroamérica».

Las inquietudes de Beltrán (2017a) tienen pertinencia, son una especie de crónica anunciada porque el mensaje final de la reunión fue justo la preocupación de Estados Unidos por el narcotráfico y la migración, con un tratamiento que privilegia

2. Se trata de una iniciativa que duró 12 años (2000-2012) con un costo de 8 mil millones de dólares, cuyos resultados, para algunos, ha sido un fracaso; si bien se reconoce que los niveles de violencia bajaron sensiblemente. «Una pregunta muy importante es si el PC es un modelo para otros países» (Rosen, 2014: 9). La percepción de éxito o fracaso depende desde dónde se mire, y al parecer para los gobiernos de Guatemala y Honduras fue un éxito, y ahora también para el gobierno de Donald Trump que está retomando la línea de la securitización. Cifras recientes indican que 2012 se registraron 49.ooo hectáreas cultivadas de coca, la más baja de la historia; sin embargo, en 2015 se llegó a 96.ooo y en 2016 a 146.000 (UNODC-Gobierno de Colombia, 2017: 23). 
el enfoque securitario. Estados Unidos ofrece migajas a los países centroamericanos a cambio de un control de sus territorios, a través de dos piezas clave: la presencia militar, con el argumento del combate al narcotráfico, y el acceso a su mercado a través de tratado de libre comercio CAFTA-RD, por sus siglas en inglés.

\section{Los contornos del ATPN: militarización y geopolítica}

El mensaje de Estados Unidos para los gobiernos centroamericanos y de México es muy claro. Rex Tillerson, en la apertura del evento expresó: «Lo que pasa en los países del Triángulo del Norte afecta directamente los intereses en seguridad y en la economía de Estados Unidos» (DPA, 17 de junio de 2017).

Por su parte, el viernes 16, en el segundo día de trabajo de la Conferencia, John Kelly, centró su atención en los temas de seguridad y expresó que «los problemas de los países centroamericanos están relacionados con la demanda estadounidense de drogas, que causa violencia devastadora en el sur, con las pérdidas de gente uniformada en México, así como periodistas y otras personas» (DPA, 17 de junio de 2017).

¿Cuáles fueron las reacciones de los participantes de esta Conferencia? Jean Manes, embajadora de Estados Unidos en El Salvador expresó que el Triángulo Norte vive un momento histórico y esperanzador. Aventuró con posibles cambios cósmicos al decir que «este es un momento histórico, sin precedentes. Las estrellas se han alineado. Esta región es un enfoque no solo de Estados Unidos, sino también de muchos otros que quieren apoyar los esfuerzos salvadoreños, guatemaltecos y hondureños» (Hernández, 21 de junio de 2017).

No hay duda de que el componente económico de la APTN juega un papel secundario frente al tema central de la seguridad. Este fue el mensaje más importante en la aludida conferencia de Miami y la pregunta que algunos plantearon fue sobre «por qué la administración de Estados Unidos y el gobierno de México están interesados en la seguridad en esta región del mundo, en Centroamérica», y la respuesta del Secretario de Seguridad fue clara: «nosotros tenemos mucho que ver con esta región» (Contreras, 17 de junio de 2017).

Por si hubiera alguna duda sobre la prioridad que tiene el gobierno de Estados Unidos en materia de seguridad, en el marco de esta conferencia los presidentes centroamericanos fueron invitados a visitar las instalaciones del Comando Sur donde «conocieron, de primera mano, el equipo y estrategias que EE. UU. utiliza para reforzar la seguridad fronteriza [...] para analizar la posibilidad de aplicarla en las fronteras del Triángulo Norte.» (Contreras, 17 de junio de 2017). 
Previa a la Conferencia, pero articulada a esta, se llevó a cabo en Cozumel, Quintana Roo, México, la Conferencia de Seguridad de Centroamérica 2017-CENTSEC, 2017-. En esta reunión realizada los días 24 y 25 de abril, se anunció la instalación de una fuerza de Tarea del Comando Sur de Estados Unidos en el departamento de Petén, Guatemala, en colaboración de los gobiernos de México y Guatemala. En este espacio fronterizo de Guatemala operan madereros, ganaderos, narcotraficantes y compañías con enormes extensiones de tierra dedicadas al cultivo de la palma africana. ${ }^{3}$

De acuerdo con Dolia Estévez, para el Pentágono, la V Conferencia de Seguridad en Centroamérica-CENTSEC 2017-, «que ha sido catalogada de "cuatro estrellas" por el alto rango de los asistentes, tiene un significado histórico ya que nunca México había aceptado ser sede» (Estévez, 21 de abril de 2017). Además, refiere que las declaraciones de la General Robinson, jefa del Comando Norte, que hiciera ante el Senado, giraban en el sentido de considerar «como un gran logro el que por primera vez en la historia institucional México aceptó copatrocinar el CENTSEC», enfatizando que de esta manera se «fortalecerá el liderazgo regional de las fuerzas armadas mexicanas en todo Centroamérica y catalizará una mayor participación de los mexicanos en temas de seguridad regional».

Por otra parte, en un escueto comunicado de prensa fechado el 25 de abril de 2017, la Secretaría de la Defensa Nacional de México informa lo siguiente:

Las secretarías de la Defensa Nacional y de Marina, dan a conocer a la opinión pública, que concluyó la Conferencia de Seguridad Centroamericana 2017 (CENTSEC 2017), la cual estuvo encabezada por el General Salvador Cienfuegos Zepeda, Secretario de la Defensa Nacional y el Almirante Vidal Francisco Soberón Sanz, Secretario de Marina, acompañados de los comandantes de los Comandos Norte y Sur de los Estados Unidos de América.

CENTSEC 2017 fue realizada por primera ocasión en México como un evento inédito, bajo un formato basado en la problemática de los países centroamericanos y en donde se llevó un diálogo estratégico para el fortalecimiento de la cooperación.

3. Es un departamento con un historial complejo, tierra de colonización sometida a un intenso proceso de extracción de sus riquezas forestales y petroleras. Fue tierra de enfrentamientos entre la guerrilla y fuerzas del ejército guatemalteco, ahora dominada y disputada por grupos de poder, articulados con sectores del gobierno guatemalteco. En los últimos años por lo menos han ocurrido dos masacres, una de ellas, en la finca los Cocos, La Libertad, en mayo de 2011, que llevó al gobierno de Álvaro Colom a tomar la decisión de implementar el Estado de Sitio por 30 días; la otra el 9 de febrero de 2014 en San Luis Petén. 
Las conclusiones se centraron en la importancia del intercambio de información, basado en la confianza, como una necesidad para responder de manera efectiva a las amenazas. Se convocó a una mayor integración de naciones para hacer eficientes las estructuras de seguridad regional; de igual manera, se enfatizó en que la desigualdad en las capacidades operativas y logísticas será un desafío a enfrentar en el corto plazo para lograr resultados en beneficio de la región (SEDENA, 2017, cursivas añadidas).

Previo a esta cumbre militar, y como otro dato indicativo del interés del gobierno de Donald Trump por reforzar las fronteras mediante la presencia de militares, funcionarios de México se reunieron con la embajadora de Estados Unidos, Kurt Tidd, jefe del Comando Sur, y la comandante Lori Robinson, del Comando Norte, entre otros. Esta reunión tuvo lugar en la Ciudad de Tapachula, Chiapas, el 31 de enero de 2017, en el espacio del antiguo aeropuerto Internacional, que ahora es el hangar del $4^{\circ}$ batallón Aeronaval de la XIV Zona Naval. Una reunión negada por las autoridades de México, pero confirmada por Estados Unidos.

En este sentido, John Kelly, secretario de Seguridad Nacional, ha sido muy claro. En una reunión organizada a principios del mes de mayo de 2017 por el grupo Atlantic Council, donde este presentó un informe, precisó: «la protección de nuestras fronteras en el sur comienzan a 15 mil millas de distancias» («John Kelly: Podemos...»), refiriéndose a Centroamérica y la frontera sur de México. Esta afirmación no es una novedad, es la reiteración de lo que se ha vivido después de los atentados de 11 de septiembre de 2001, ahora con mayor contundencia y con mayor colaboración del gobierno de México.

Así mismo, debemos recordar que la iniciativa CARSI, la versión centroamericana de la Iniciativa Mérida, viene operando desde 2008. El Comando Sur en Centroamérica ha venido actuando a través de la Iniciativa CARSI y en este marco se instrumentó la Operación Martillo, que funciona en colaboración con otros países. John Kelly, entonces jefe de este comando, ha reiterado el éxito de la operación, las cifras de incautación de droga lo ejemplifican: desde enero de 2012, cuando comenzaron las acciones de la Operación, en año y medio, se habían decomisado más de 300 mil libras de cocaína y más de 25 libras de marihuana. También se incluye el decomiso de 159 buques y aeronaves, la detención de 507 personas y la interrupción de 222 toneladas métricas de cocaína (Panting, 3 de septiembre de 2013).

La presencia del Comando Sur ha sido una constante, en particular se indica la importancia que ha tenido la colaboración con el gobierno de Honduras. En 2013, el director de asuntos públicos de dicho comando, Greg Julian, fue enfático en señalar que: 
Honduras es clave en la lucha contra el narcotráfico por su ubicación y por el trabajo conjunto que hacemos. Sabemos que los vuelos que dejaron de llegar a República Dominicana encontraron una nueva ruta dirigiéndose a suelo hondureño.

La colaboración de las autoridades hondureñas ha sido fantástica y, aunque sabemos que tanto ellos como nosotros tenemos limitaciones de presupuesto, ponemos todo nuestro empeño en aprovechar al máximo lo que tenemos disponible. Honduras tiene un papel super importante en la lucha contra el narcotráfico porque la mayoría del tráfico pasa por Centroamérica (Panting, 3 de septiembre de 2013).

En ese contexto, el 31 de marzo de 2016 se anunció la reconversión de la base militar de Palmerola, Honduras, en aeropuerto civil, reemplazando al que se encuentra ubicado en la capital, Tegucigalpa. La base fue construida por el gobierno de Washington a mediados de la década de los 80, en el marco de la guerra civil en la región, alberga un promedio de 500 soldados estadounidenses de la Fuerza de Tarea Conjunta Bravo, del Comando Sur. Su costo se calculó en 163 millones de dólares, su construcción comenzaría en junio de 2016 -en realidad comenzó en diciembre(«Honduras: Inician construcción...») y su conclusión en enero de 2018. Una concesión por 30 años mediante la modalidad de alianza público-privado. El presidente Juan Orlando Hernández ha dicho que Palmerola seguirá siendo una base militar.

La declaración del presidente hondureño confirma la importancia que tiene esta base para las operaciones del gobierno de Estados Unidos en la región del Caribe, incluso en una eventual intervención militar en Venezuela, ${ }^{4}$ como se ha afirmado en un documento (Operación Venezuela Freedom-2) firmado supuestamente por el Almirante Kurt W. Tidd, comandante del Comando Sur, fechado el 26 de febrero de 2016, traducido y reproducido por diario El Libertador. El citado documento hace 12 recomendaciones, en la última habla de una estrategia de intervención mediante fuerzas especiales destacamentadas en la base militar de Palmerola, Honduras, con apoyo de las bases militares de las islas antillanas de Aruba y Curazao, así como las colombianas de Larandia, Tres Esquinas, Puerto Leguízamo y Leticia.

Del 1 al 3 de abril, en la ciudad de Guatemala, se llevó a cabo la Conferencia Regional de Seguridad Centroamericana 2014 -CENTSEC 2014-. En el marco de este evento se actualizó CARSI ${ }^{5}$ y se habló, de nueva cuenta, de los éxitos de la

4. Las recientes declaraciones del presidente Trump confirman estas sospechas: «Tenemos muchas opciones para Venezuela, incluyendo una posible opción militar si es necesario» (Brooks, 2017).

5. Esta consistió en la ampliación «de asistencia en seguridad que involucra a agencias gubernamentales, socios internacionales y la ayuda de los sectores público y privado» (Fiona, 7 de abril de 2014). 
Operación Martillo: ${ }^{6}$ «entre el lanzamiento de la Operación Martillo en enero de 2012 y finales de enero del 2014, la iniciativa confiscó $278.611 \mathrm{~kg}$ de cocaína y 27.556 $\mathrm{kg}$ de marihuana durante 444 eventos que condujeron a 620 arrestos y al decomiso de 205 naves» (Fiona, 7 de abril de 2014).

Especial atención ha tenido el Comando Sur en Centroamérica a partir de 2013. El 29 de abril de ese año, en comparecencia ante el Congreso, 7 John Kelly, jefe del Comando Sur, expresó que «reubicó su enfoque a instituciones de seguridad centroamericanas involucradas en las misiones adecuadas de defensa, tales como seguridad marítima y fronteriza» (Luxner, 30 de abril de 2014). De la misma manera, al referirse a la Operación Martillo dijo que ésta «sigue mostrando el compromiso de los Estados Unidos, nuestros países socios y los aliados europeos para contrarrestar la expansión de las organizaciones delictivas transnacionales y proteger a los ciudadanos en Centroamérica de la violencia, el daño y la explotación provocadas por las redes delictivas» (Luxner, 30 de abril de 2014).

Por su parte, Luís Arreaga, Vicesecretario de Estado de EE. UU. para asuntos internacionales de narcóticos y cumplimiento de la ley, expresó: «no se equivoquen, va a llevar muchos años más para ver los frutos de nuestros esfuerzos en Centroamérica debido a la magnitud de las amenazas contra el estado de derecho y las instituciones creíbles del gobierno» (Luxner, 30 de abril de 2014).

Otro antecedente importante de la presencia del Comando Sur en Centroamérica es el apoyo al establecimiento del Grupo de Alto Nivel de Seguridad y Justicia entre Honduras y Guatemala, formalizado el 23 de marzo de 2005, con lo que entra en vigor las operaciones de la Fuerza de Tarea Binacional Maya-Chortí («Presidentes de Honduras...») con integrantes de los ejércitos y policías de ambos países, y equipo proporcionado por Estados Unidos.

La referencia a estos hechos previos a la Conferencia de Miami tiene sentido porque son indicativos de la tendencia de los intereses de Estados Unidos en la región, que no son novedad, pero ahora aparece con mayor contundencia el énfasis militar y policial, rasgo característico del gobierno de Donald Trump.

Vale la pena recordar que cuando inició la crisis de los niños migrantes (julio de 2014), los presidentes de Guatemala, Otto Pérez Molina, y de Honduras, Juan Orlando Hernández, viajaron a Washington y pidieron replicar el Plan Colombia en sus países. En esa ocasión Pérez Molina expresó que los países centroamericanos

6. Participan Canadá, Belice, Colombia, Costa Rica, El Salvador, Francia, Guatemala, Honduras, Países Bajos, Nicaragua, Panamá, España, Reino Unido, Estados Unidos.

7. Esta reunión se denominó «de cara al narcotráfico transnacional: una evaluación de las sociedades regionales». 
tienen responsabilidades, pero «si estamos acompañados con un plan de Centroamérica como ya se hizo en el Plan Colombia u otros planes de Estados Unidos no tengo la menor duda que estaríamos resolviendo de raíz los problemas que están en la frontera» («Honduras y Guatemala piden...»).

Dos semanas después de la petición de los presidentes centroamericanos la respuesta del gobierno de Estados Unidos fue negativa. El vicepresidente Joe Biden cuestionó «¿por qué no tenemos un Plan Colombia para Centroamérica?» y la respuesta fue precisa «porque los gobiernos centroamericanos no están ni cerca de estar preparados para tomar la clase de decisiones que tomaron los colombianos [...] Son decisiones muy duras, domésticamente muy difíciles» («EE.UU. descarta un "Plan Colombia"...»).

Todo parece indicar que la administración de Trump considera que ahora sí es posible aplicar un Plan Colombia centroamericano. En su discurso inaugural de la conferencia de Miami, Tillerson así lo dejó ver:

[...], buscamos reforzar la seguridad nacional de Estados Unidos, asegurar nuestras fronteras y promover el interés económico de Estados Unidos. Promover la prosperidad en Centroamérica es un componente clave de este esfuerzo, ya que nuestros intereses de prosperidady seguridad están estrechamente vinculados mediante el movimiento de ideas, personas y bienes (Tillerson, 2017; cursivas añadidas).

Lo que se puede leer en este discurso es una actualización del interés geopolítico de la administración Trump por Centroamérica, pero sobre todo los tres países que integran el llamado Triángulo Norte. Al respecto, Tillerson refirió: «una América Central segura y próspera contribuye a una mayor seguridad y prosperidad en Estados Unidos y el Hemisferio Occidental» (Tillerson, 2017).

La estrategia de la Casa Blanca sigue su marcha, nada ni nadie parece oponerse, todo lo contrario: con el propósito de dar seguimiento a los acuerdos de la conferencia de Miami, entre México y Estados Unidos, el secretario de seguridad interna, John F. Kelly ${ }^{8}$ estuvo en México del 5 al 7 de julio de 2017 y se reunió con el presidente Peña Nieto en la residencia oficial de Los Pinos. Acompañados, por la parte mexicana, del embajador en Washington Gerónimo Gutiérrez y el canciller Luis Videgaray; por Estados Unidos la embajadora en México Roberta Jacobson, el director de la CIA Michael Richard Pompeo, y el senador por Arkansas Tom Cotton. Los temas abordados fueron migración, seguridad, fronteras; según lo trascendido

8. El 28 de julio de 2017 fue nombrado nuevo jefe de gabinete de la Casa Blanca, en sustitución de Reince Prebius, el presidente escribió en su cuenta de Twitter: «Tengo el placer de informar que he nombrado al general y secretario John Kelly como jefe del gabinete de la Casa Blanca» («John Kelly es nombrado...»). 
en los medios de comunicación el presidente Peña Nieto se refirió a la Conferencia sobre Prosperidad y Seguridad en Centroamérica «como ejemplo de éxito de esfuerzos para enfrentar los retos y aprovechar las oportunidades de la región en materia económica, migratoria y seguridad» (Jiménez y García, 6 de julio de 2017).

Kelly se reunió, por separado, con los secretarios Luis Videgaray y José Antonio Meade, así como con los secretarios de Marina Vidal Francisco Soberón, y de la Defensa Nacional, Salvador Cienfuegos; el último día lo hizo con el secretario de gobernación, Miguel Ángel Osorio Chong. En estas reuniones los temas abordados fueron el combate del tráfico de drogas a Estados Unidos y la seguridad. El funcionario estadounidense, junto con los secretarios de Marina y Defensa Nacional, sobrevolaron zonas de incidencia de cultivo de amapola, y observó la destrucción de cinco plantíos (Aranda y Castillo, 7 de julio de 2017).

De las palabras del secretario Kelly podemos recuperar dos señalamientos que reflejan el tipo de relaciones que está asumiendo el gobierno de México con Estados Unidos. Primero: «reconocemos que la prosperidad y la seguridad están entrelazados, y las redes criminales siguen creciendo con su influencia. Si nuestros dos países no trabajan juntos, van a ganar poder»; segundo: «México ha tomado el liderazgo en el desarrollo económico y el Triángulo Norte (sic), y acaba de organizar la Conferencia sobre la Prosperidad y la Seguridad para Centroamérica, junto con Estados Unidos» (Kelly, 2017). Estas revelaciones confirman que México jugará un papel más activo del que ya tenía en el seguimiento de la APTN, ahora con énfasis en la seguridad.

Estados Unidos tiene todos los instrumentos para someter a los gobiernos de los países centroamericanos: comercio, a través del Tratado de Libre Comercio CAFTA-RD-, inversión extranjera directa, asistencia al desarrollo-USAID-, deuda externa, migración, entre otros. En el tema migratorio la administración Trump está considerando la posibilidad de suspender ${ }^{9}$ o limitar el estatus de protección temporal -TPS, por sus siglas en inglés-. El país beneficiario del TPS es definido por la secretaría de Seguridad Nacional de acuerdo con ciertos criterios como la existencia de una guerra civil o conflicto armado, desastres naturales y otras circunstancias extraordinarias. ${ }^{10} \mathrm{El}$ primer país beneficiado fue Nicaragua, como respuesta a los que emigraron a Estados Unidos en el marco del conflicto armado; en el caso de El Salvador, el TPS fue otorgado en 2001, luego en Guatemala y otros países.

9. El 6 de noviembre el Departamento de Seguridad Interna tomó la decisión de no renovar el TPS para Nicaragua.

10. El TPS se inscribe en la llamada Ley NACARA (Nicaraguan Adjustmen and Central American Relief) aprobada por el Congreso de Estados Unidos en 1997, durante la administración del presidente Bill Clinton. 
Se estima que actualmente (2017) existen 580 mil migrantes centroamericanos y haitianos bajo el estatus de protección temporal; dentro de este grupo se registran 100 mil hondureños y 190 mil salvadoreños. Los gobiernos de la región no tienen la capacidad de reinsertar a la vida productiva a estos migrantes, de producirse una eventual deportación.

Cada año los gobiernos de la región negocian la prórroga, pero ahora con el gobierno de Trump se ha filtrado la amenaza sobre la posibilidad de eliminar el TPS para los haitianos, situación que ha provocado inquietud entre los centroamericanos; por lo mismo el presidente salvadoreño, Salvador Sánchez Cerén, pidió a Michael Bennet, senador demócrata, promover el TPS. En un escenario de cancelación de este mecanismo, los países de la región no tendrían capacidad para recibir y dar opciones de empleo a los deportados, de ahí que el TPS se convierte en un instrumento de presión de Estados Unidos para que los países centroamericanos lleven a cabo las políticas que interesan al país del norte.

\section{La prosperidad ¿para quién?}

La «prosperidad» ha sido eje de los discursos con que los gobiernos neoliberales han vendido la idea del «libre comercio», de la apertura de mercados, de las concesiones mineras y la explotación de recursos estratégicos que poseen los países subdesarrollados, todo esto aderezado de políticas de seguridad frente a cualquier movimiento social o de grupo que se oponga a tales políticas. La «prosperidad», sin embargo, no aplica para la movilidad humana, sobre todo para la migración en condiciones irregulares. Los migrantes no tienen derecho a ser «prósperos», a pesar de la importancia estratégica que representa el trabajo vivo para la valorización del capital; solo el trabajo muerto, cristalizado en mercancías, puede transitar.

El discurso de la «prosperidad» comenzó a manejarse profusamente después de la firma del Acuerdo para la Seguridad y Prosperidad de América del Norte-ASPAM- ${ }^{11}$ que algunos analistas denominaron TLCAN Plus en tanto que el TLCAN había llegado a la desgravación total en los intercambios comerciales. La prédica de la «prosperidad» esconde el propósito esencial que consiste en proveer seguridad a Estados Unidos mediante el resguardo de las fronteras con Canadá y México, que se extiende a Centroamérica y el Caribe. El marco en el que se comprende mejor este propósito subyacente son los atentados del 11 de septiembre de 2001, y todas las secuelas en materia de seguridad interior y hemisférica, el acuerdo sobre Fronteras 
Inteligentes surgido a partir de la cumbre de Monterrey en marzo de 2002, así como la Iniciativa para la Administración de la Frontera en el Siglo XXI de 2010, que a su vez constituye el segundo pilar de la Iniciativa Mérida.

El mito neoliberal del progreso ha quedado refutado por la brutalidad de la actuación del capital que se expresa en la destrucción del ambiente, la concentración de tierras y del capital, el desempleo ${ }^{12}$ y la precarización laboral, ${ }^{13}$ la pobreza y exclusión de millones de seres humanos, que finalmente se articula con la migración laboral de carácter irregular. Este es el verdadero significado de la «prosperidad» en clave neoliberal.

La crisis del sistema migratorio, en el marco de la nueva era del capitalismo, dominada por el capital financiero, devela los variados mecanismos para detener la migración en momentos de recesión y crisis económica a fin de «equilibrar» la oferta de fuerza de trabajo. En este sentido, Foucault refería que «el ajuste entre la acumulación de hombres y la del capital, la articulación entre el crecimiento de los grupos humanos y la expansión de las fuerzas productivas y la repartición diferencial de la ganancia, en parte fueron posibles gracias al ejercicio del bio-poder en sus formas y procedimientos múltiples» (1998: 72). Esta idea se redimensiona en el nuevo escenario, donde por métodos punitivos se interrumpe el «libre juego de la oferta y la demanda». Es una migración deseada en momentos de crecimiento económico y rechazada en tiempos de recesión. Pero como bien indica el citado autor, el dominio de los cálculos de la biopolítica no implica el control absoluto, «no significa que la vida haya sido exhaustivamente integrada a técnicas que la dominen o administren; escapa de ellas sin cesar» (1998: 74).

Sin ser demasiado prolijo, se puede decir que, frente a las acciones de los gobiernos de México y Estados Unidos, los migrantes evaden, buscan nuevos mecanismos, nuevas rutas que les permitan llegar a su destino, desde luego con un elevado costo financiero y sufrimiento. El enfoque biopolítico de la migración, introducido en los programas de contención como el APTN y el Programa Fronteras sur, se traduce en acuerdos específicos entre los gobiernos de la región, México y Estados Unidos, como la creación de una base de datos biométricos para el registro de migrantes aprehendidos, que viola la resolución de la ONU 45/34 sobre la Convención Inter-

12. «El desempleo es con toda seguridad el riesgo social más grave hoy en día, el que tiene los efectos desestabilizadores y desocializantes más destructores para quienes lo sufren» (Castel, 1997: 459).

13. Estamos de acuerdo con Isabell Lorey cuando refiere que «La precarización significa más que puestos de trabajo inseguros, más que una cobertura social insuficiente dependiente del trabajo asalariado. En tanto que incertidumbre y exposición al peligro, abarca la totalidad de la existencia, de los cuerpos, los modos de subjetivación. Es amenaza y constricción, al mismo tiempo que abre nuevas posibilidades de vida y de trabajo. La precarización significa vivir con lo imprevisible, la contingencia. (Lorey, 2016: 17). 
nacional sobre la Protección de los Derechos de todos los Trabajadores Migrantes y sus Familias. El bio-poder se manifiesta en la concepción que el gobierno de Estados Unidos tiene sobre el fenómeno migratorio, que impone sobre el resto de los gobiernos de los países centroamericanos y México.

A pesar de todo, en Estados Unidos hay otras voces que difieren de un enfoque militar o exclusivamente militar. En este sentido el Atlantic Council en un documento reciente apunta en una de sus conclusiones la siguiente idea:

No hay una bala mágica para todos los problemas que asolan el Triángulo Norte hoy. Cualquier estrategia que se centra sólo en la seguridad y descuida el fortalecimiento institucional y el fomento de un clima económico propicio será inevitablemente insuficiente. Sólo un enfoque holístico, que se basa en los esfuerzos recientes, pero también reconozca sus deficiencias y empuje para una acción más asertiva, generará cambios transformacionales. Es decir, lo que se necesita para profundizar el curso en la región. Si no, estamos condenados a un deterioro continuo de la economía local, el Estado de derecho y la seguridad con reverberaciones no sólo localmente sino no también al norte del Río Bravo (Atlantic Council, 2017:38).

Los tres problemas que preocupan al gobierno de Estados Unidos (migración, narcotráfico, seguridad fronteriza) requieren, efectivamente, otra mirada que no sea la que enfatizó la Conferencia de Miami marcada por la biopolítica y la necropolítica. La migración no se ha detenido y no se va detener con más medidas punitivas, como lo muestran las cifras de la Patrulla Fronteriza (cuadros 1 y 2). La solución pasa por una revisión profunda de la situación de la región y de la instrumentación de medidas de corto, mediano y largo plazos para restaurar las condiciones básicas de convivencia, de garantía de los derechos humanos, los derechos económicos, sociales y políticos.

Como se puede observar en el cuadro 2, después del férreo control de las fronteras, a partir del verano de 2014, y en las rutas de tránsito, se produjo una reducción en el número de capturas; sin embargo, en 2016 las cifras volvieron a subir al grado de equipararse a las de 2014 que dispararon la crisis. Es posible que el año fiscal 2017 cierre con alrededor de 45.0oo, lo que confirmaría la hipótesis de que no es posible detener la migración con medidas punitivas. Esto hace que el gobierno de Estados Unidos mantenga la preocupación por el tema migratorio, y el papel que debe cumplir el gobierno de México en el control. 


\begin{tabular}{l|l} 
CUADRO 2 & $\begin{array}{l}\text { Aprehensión de niños no acompañados por país en la frontera sur } \\
\text { de Estados Unidos (Años fiscales 2013-2016 y año fiscal } 2017 \text { hasta } \\
\text { el 31 de agosto) }\end{array}$
\end{tabular}

\begin{tabular}{|l|c|c|c|c|c|}
\hline País / año & $\mathbf{2 0 1 3}$ & $\mathbf{2 0 1 4}$ & $\mathbf{2 0 1 5}$ & $\mathbf{2 0 1 6}$ & $\mathbf{2 0 1 7 ^ { * }}$ \\
\hline El Salvador & 5.990 & 16.404 & 9.389 & 17.512 & 8.850 \\
\hline Guatemala & 8.068 & 17.057 & 13.589 & 18.913 & 13.594 \\
\hline Honduras & 6.747 & 18.244 & 5.409 & 10.468 & 7.314 \\
\hline México & 17.240 & 15.674 & 11.012 & 11.926 & 8.056 \\
\hline Total & $\mathbf{3 8 . 0 4 5}$ & $\mathbf{6 7 . 3 7 9}$ & $\mathbf{3 9 . 3 9 9}$ & $\mathbf{5 8 . 8 1 9}$ & $\mathbf{3 7 . 8 1 4}$ \\
\hline
\end{tabular}

${ }^{*}$ Cifras hasta el 31 de agosto, el año fiscal concluye en septiembre. Fuente: CBP, 2017.

CUADRO 3 | Unidades familiares ${ }^{\star}$, aprehensiones por país, años fiscales 2016-2017

\begin{tabular}{|c|c|c|}
\hline País / año & 2016 & $2017^{* *}$ \\
\hline El Salvador & 27.114 & 23.439 \\
\hline Guatemala & 23.067 & 22.750 \\
\hline Honduras & 20.226 & 20.990 \\
\hline México & $3 \cdot 481$ & 2.107 \\
\hline Total & 73.888 & 69.286 \\
\hline
\end{tabular}

* La unidad familiar representa el número de individuos, menores de 18 años con padre o tutor legal, aprehendidos con los miembros de la familia. ${ }^{* *}$ Cifras hasta el 31 de agosto, el año fiscal concluye en septiembre. Fuente: CBP, 2017. 
Lo mismo se puede ver en el cuadro 3, donde las cifras no distan mucho de un año fiscal a otro, sobre todo si tomamos en cuenta que falta un mes para la conclusión del año fiscal 2017. La suma de ambos cuadros retrata una realidad muy distinta a lo que han venido proyectando los programas implementados para detener la migración-Iniciativa para la Prosperidad y Programa Frontera sur-: solo han elevado el costo económico, social y humano. En este sentido, resulta muy interesante un fragmento de una conversación con un experimentado coyote hondureño: «Aquí nadie va a parar esto. Las fronteras siempre se pondrán peor, siempre serán duras. Mientras no pares el hambre y la violencia aquí, la gente se va a seguir yendo» (Ávila et.al., 2017). En este marco, la «industria de la migración» se ha fortalecido, el cobro por llevar a una persona hasta Estados Unidos es muy redituable, en estos tiempos difíciles, las tarifas son de 8.500 dólares por adulto y 4.00o dólares por niño, la red de coyotaje está bien establecida, al respecto el entrevistado dice: «a veces la gente se asusta porque se nos ponen dos camionetas y se bajan hombres armados. Chequean que vayan las personas que reporté, no puedo engañarlos: si seis personas llevo, seis reporto. Le pago al patrón US\$9oo por cada uno» (Ávila et. al., 2017).

Por otra parte, el narcotráfico hasta ahora no se ha detenido, a pesar de las cifras oficiales sobre incautación, porque la demanda en Estados Unidos sigue creciendo, y a pesar de la promesa de levantar el muro siempre habrá forma de infiltrar el resguardo fronterizo en la medida en que se requiera. Son problemas sistémicos que requieren un enfoque distinto que implica un control de oferta y demanda, de producción y consumo, del circuito de distribución y de las redes financieras. Hay muchos intereses mezclados en los asuntos del narcotráfico y del control de las fronteras, incluyendo los grandes fabricantes y proveedores de armas y equipos a los gobiernos involucrados. Los países centroamericanos no tienen la capacidad técnica, financiera e institucional para hacer frente a estos desafíos; en todo caso son víctimas en su propio territorio de una doble situación, las redes de narcotráfico y la presión de Estados Unidos.

El enemigo no son los migrantes que buscan salir de la miseria a que los ha llevado el sistema. El enemigo es el sistema económico que los ha expulsado; es el sistema que protege al capital pero no a la sociedad; es el sistema que ha permitido un esquema de distribución del ingreso profundamente desigual y ha empobrecido a millones de personas que buscan el «progreso» abandonando su lugar de origen. Un sistema que debe ser revisado en todas sus partes y componentes para reestructurarlo. Obviamente, ningún gobierno quiere tocar el sistema porque implicaría afectar seriamente intereses de poderosos grupos nacionales y extranjeros que se 
han beneficiado. Por el contrario, se sigue alentando con planes como la APTN, ahora con un enfoque más de seguridad.

¿De qué prosperidad estamos hablando, cuando se cierran las fronteras a la migración laboral? Vivimos tiempos de brutalidad como dice Sassen (2015), pero se nos hace creer que vamos hacia un mundo feliz. Se trata de lo que Franco Berardi denomina «ideología virtual», definida como «una mezcla de futurismo tecnológico, evolucionismo social y neoliberalismo económico» (2003: 9). Como ocurrió con el desarrollo, el mito de Prometeo, la prosperidad ahora es «la promesa de la felicidad y la autorrealización en el trabajo» (Berardi, 2003). Esto que se encuentra en el imaginario de la new economy (modelo productivo y discurso cultural) que se derrumbó con la crisis financiera. Sin embargo, a pesar de haber pasado por sucesivas crisis, el discurso se sigue vendiendo porque hay gobiernos que lo compran, como ha venido ocurriendo en los países centroamericanos.

\section{Reflexión final}

Toda Centroamérica, especialmente los países del llamado Triángulo Norte, enfrenta una compleja problemática donde se articula pobreza, pandillas, crimen transnacional, narcotráfico y un modelo económico despiadado que destruye las formas de socialización y expulsa población. En estas condiciones: ¿es posible pensar en que las medidas adoptadas para hacer frente a la migración, el narcotráfico, y generar «prosperidad», son las adecuadas?

La llamada Iniciativa para la Prosperidad es una pálida sombra frente a las dificultades estructurales, incluso si se implementara un «Plan Colombia para Centroamérica», que ha comenzado a dibujarse en la administración del presidente Trump. En dos años y medio (de noviembre de 2014 hasta junio de 2017) la realidad muestra que el estado de cosas no ha cambiado con la implementación de la iniciativa, aunque los gobiernos de los tres países centroamericanos se ufanen en mostrar el cambio en sus indicadores de violencia. Quizá la presión de los menores migrantes se haya detenido un poco, así lo hacen ver las cifras oficiales de detenciones tanto en México como en Estados Unidos, pero el problema no está resuelto, y no podrá resolverse mientras no se toquen las causas estructurales.

Profundizar en una estrategia securitaria como muestra el relanzamiento de la Iniciativa para la Prosperidad en la Conferencia de Miami, llevaría a una situación de mayor tensión, se produciría más violencia, desplazamiento de población, y en última instancia tampoco resolvería el problema del narcotráfico como no lo resolvió en Colombia. Por el contrario, la nueva estrategia significa mayor intervención de 
Estados Unidos, mayor control de la región centroamericana, el sur de México y el Caribe mediante la presencia de tropas combinadas estadounidenses y de los países de la región, con la colaboración de México.

No debemos engañarnos, desde los atentados del 11 de septiembre del 2001 la cuestión de la seguridad interior, fronteriza y hemisférica se convirtió en una prioridad para el gobierno de Estados Unidos. Lo que hoy vocifera el presidente Trump es una máscara que oculta el proceso seguido desde hace casi dos décadas, que se concreta en el avance en la construcción del muro físico, de la vigilancia electrónica, la instalación de sensores remotos y la permanencia de aviones no tripulados, además del incremento progresivo de los elementos de la Patrulla Fronteriza, reforzado con efectivos de la Guardia Nacional.

\section{Bibliografía}

ARANDA, J.y CASTILLO, G. (7 de julio de 2017). Sobrevuela Kelly áreas de cultivo de droga en Guerrero. La Jornada. Recuperado de:http://www.jornada.unam.mx/2017/07/o7/politica/oo5n1pol

ATLANTIC COUNCIL, (2017). Building a Better Future. A Blueprint for Central America's Nothern Triangle. Washington, DC. Recuperado de: http://publications.atlanticcouncil.org/northern-triangle/assets/AC_Northern_Triangle.pdf

ÁVILA, J., MADARIAGA, H. y ANDINO, J. (5 de mayo de 2017). El miedo a Trump es temporal; la migración sigue. Plaza Pública. Recuperado de: https://www.plazapublica.com.gt/content/ el-miedo-trump-es-temporal-la-migracion-sigue

BELTRÁN, A. (22 de diciembre de 2015). ¿Qué aprobó el Congreso Estadounidense para Centroamérica en el presupuesto 2016? Wola. Recuperado de: https://www.wola.org/es/analisis/ que-aprobo-el-congreso-estadounidense-para-centroamerica-en-el-presupuesto-de-2016/

BELTRÁN, A. (2017a). Preocupaciones claves sobre la cumbre de Centroamérica en Miami. Wola. Disponible en: https://www.wola.org/es/analisis/preocupaciones-claves-sobre-cumbre-de-centroamerica-en-miami/

BELTRÁN, A. (2017b). ¿Qué incluye el presupuesto estadounidense del 2017 para Centroamérica? Wola. Recuperado de:https://www.wola.org/es/analisis/que-incluye-el-presupuesto-estadounidense-del-2017-para-centroamerica/

BERARDI, F. (2003). La fábrica de la infelicidad. España. Traficantes de Sueños.

BROOKS, D. (12 de agosto de 2017). Amenaza Trump con intervenir militarmente en Venezuela. La Jornada. Recuperado de: http://www.jornada.unam.mx/2017/o8/12/mundo/o2on1mun 
Camilo Atala: Empresarios debemos ser aliados del Plan para la Prosperidad. (26 de febrero de 2015). La Prensa. Recuperado de: http://www.laprensa.hn/honduras/808749-417/camilo-atala-empresarios-debemos-ser-aliados-del-plan-para-la-prosperidad

Cancilleres llevan borrador de plan Alianza a Joe Biden. (15 de marzo de 2015). La Prensa. Recuperado de: http://www.laprensa.hn/mundo/822701-410/cancilleres-llevan-borrador-de-plan-alianza-a-joe-biden

CASTEL, R. (1997). La metamorfosis de la cuestión social. Argentina. Paidós.

CBP, (2017). U.S. Border Patrol Southwest Border apprenhsions by sector. Disponible en: https:// www.cbp.gov/newsroom/stats/usbp-sw-border-apprehensions

Comando Sur tiene en sus planes usar Honduras para atacar Venezuela. (26 de febrero de 2016). El Libertador. Recuperado de: http://www.web.ellibertador.hn/index.php/noticias/internacionales/1325-comando-sur-tiene-en-sus-planes-usar-honduras-para-atacar-venezuela

CONTRERAS, G. (16 de junio de 2017 ). EE. UU. recalca a Centroamérica: la seguridad es primero. Prensa Libre. Recuperado de: http://www.prensalibre.com/guatemala/politica/gobiernos-de-eeuu-mexico-y-centroamerica-discuten-temas-de-seguridad-regional

DPA. (17 de junio de 2017). Frases más destacadas que dejan la cumbre sobre Centroamérica. Prensa Libre. Recuperado de: http://www.prensalibre.com/internacional/frases-mas-destacadas-que-deja-la-cumbre-sobre-centroamerica

EE. UU. descarta un «Plan Colombia» en Centroamérica. (7 de agosto de 2014). Prensa Libre. Recuperado de: http://www.prensalibre.com/internacional/biden-plan_colombia-centroamerica_O_1188481335.html?print=1

ESTÉVEZ, D. (21 de abril de 2017). Cumbre militar en Cozumel, escenario del debut de México como líder regional. SinEmbargo. Recuperado en: http://www.sinembargo.mx/21-04-2017/3197684

FIONA, A. (7 de abril de 2014). Conferencia regional de seguridad evalúa Operación Martillo. Diálogo. Guatemala. Recuperado de: http://dialogo-americas.com/es/articles/rmisa/narconews/ features/main/2014/04/o7/feature-O1

FOUCAULT, M. (1998). Historia de la sexualidad, vol. I. México. Siglo XXI Editores.

HERNÁNDEZ, A. (21 de junio de 2017). Triángulo Norte vive un momento histórico y esperanzador con Trump. La Prensa Gráfica. Recuperado de: http://www.laprensagrafica.com/2017/o6/21/ triangulo-norte-vive-un-momento-historico-y-esperanzador-con-trump

Honduras estudia ser sede para Fuerza Especial de EUA para Latinoamérica. ( 7 de abril de 2015). La Prensa. Recuperado de: http://www.laprensa.hn/honduras/828531-410/honduras-estudia-ser-sede-para-fuerza-especial-de-eua-para-latinoam\% $\mathrm{C}_{3} \%$ A9rica?utm_source=laprensa. hn\&utm_medium=website\&utm_campaign=noticias_relacionadas 
Honduras y Guatemala piden a EUA un «Plan Colombia» para la región. (24 de julio de 2014). La Prensa. Recuperado de: http://www.laprensa.hn/inicio/732140-96/honduras-y-guatemala-piden-a-eua-un-plan-colombia-para-la-regi\% $\mathrm{C}_{3} \% \mathrm{~B}_{3} \mathrm{n}$

Honduras: Inician construcción del Aeropuerto Internacional de Palmerola. 5 de diciembre de 2016). El Heraldo. Recuperado de: http://www.elheraldo.hn/pais/10239o8-466/honduras-inician-construcci\% $\mathrm{C}_{3} \% \mathrm{~B} 3 \mathrm{n}$-del-aeropuerto-internacional-de-palmerola

Hoy es un día histórico para Centroamérica. Otto Pérez. (26 de febrero de 2015). La Prensa. Recuperado de: http://www.laprensa.hn/honduras/808923-417/hoy-es-un-d\%C3\%ADa-hist\%C3\%B3rico-para-centroam\%C3\%A9rica-otto-p\%C3\%A9rez

JIMÉNEZ, H. y GARCÍA, A. (6 de julio de 2017). EPN y EU acuerdan combatir el crimen transnacional. El Universal. Recuperado de: http://www.eluniversal.com.mx/articulo/nacion/seguridad/2017/o7/6/epn-y-eu-acuerdan-combatir-el-crimen-transnacional

John Kelly es nombrado como nuevo jefe de gabinete de la Casa Blanca, sustituye a Reince Priebus. (28 de julio de 2017). The Dominican Journal. Recuperado de: http://dominicanjournalusa. com/john-kelly-es-nombrado-como-nuevo-jefe-de-gabinete-de-la-casa-blanca-sustituye-reince-priebus/

John Kelly: Podemos hacer la diferencia en América Central. (4 de mayo de 2017). Voz de América. Recuperado en: https://www.voanoticias.com/a/inmigracion-centroamerica-atlantic-council/3837506.html

KELLY, J. (2017). «Palabras del Secretario de Seguridad Nacional John Kelly». Embajada y Consulados de Estados Unidos en México. Disponible en: https://mx.usembassy.gov/es/palabras-del-secretario-de-seguridad-nacional-john-kelly/

LOREY, I. (2016). Estado de inseguridad. Madrid. Traficantes de Sueños.

LUXNER, L. (30 de abril de 2014). Gral. John Kelly, del Comando Sur, y otros funcionarios hablan sobre el reto de las drogas. Diálogo. Recuperado de: http://dialogo-americas.com/es/articles/ rmisa/features/regional_news/2014/05/30/southcom-hearing

Marines de Estados Unidos llegarán a Colón y La Mosquitia. (14 de abril de 2015). La Prensa. Recuperado de: http://www.laprensa.hn/honduras/831111-410/marines-de-estados-unidos-llegar\% $3 \%$ A1n-a-col\%C3\%B3n-y-la-mosquitia

Monitoreando Programas de Asistencia de EE. UU. (2017). Wola. Recuperado de: https://www. wola.org/es/monitoreando-asistencia-centroamerica/

PANTING, C. (3 de septiembre de 2013). Comando Sur: Honduras, clave en la lucha contra el narcotráfico», La Prensa. Recuperado de: http://www.laprensa.hn/honduras/apertura/382707-98/ comando-sur-honduras-clave-en-la-lucha-contra-el-narcotr\% $3 \%$ A1fico 
Plan de la Alianza para la Prosperidad del Triángulo Norte. (2015). Avances 2015y el plan de 2016. Recuperado de: https://docs.wixstatic.com/ugd/f54626_3e5de1a71a8542ceb41d18195dcca6e6.pdf

Presidentes de Honduras y Guatemala lanzan Fuerza Maya-Chortí. (24 de marzo de 2015). La Prensa. Recuperado de: http://www.laprensa.hn/honduras/825080-410/presidentes-de-honduras-y-guatemala-lanzan-fuerza-maya-chort $\% \mathrm{C}_{3} \% \mathrm{AD}$

RODRÍGUEZ, D. (7 de abril de 2015). Arribo de marines de EUA a Honduras se pidió a Defensa. La Prensa. Recuperado de: http://www.laprensa.hn/honduras/828915-410/arribo-de-marinesde-eua-a-honduras-se-pidi\%C_ $3 \%$ B3-a-defensa?utm_source=laprensa.hn\&utm_medium=website\&utm_campaign=noticias_relacionadas

ROSEN, J. D. (2014). Lecciones y resultados del Plan Colombia (2000-2012). Contextualizaciones latinoamericanas. 6(10).

SASSEN, S. (2015). Expulsiones. Brutalidad y complejidad en la economía global. Argentina. Katz Editores.

SEDENA (Secretaría de la Defensa Nacional). «Concluye Conferencia de Seguridad de Centroamérica 2017». Comunicado de prensa, 25 de abril de 2017. Recuperado en: https://www.gob. $\mathrm{mx} /$ sedena/prensa/concluye-conferencia-de-seguridad-de-centroamerica-2017-104652.

TILLERSON, R. (2017). «Discurso del Secretario de Estado Rex Tillerson en la Conferencia sobre Prosperidad y Seguridad en América Central». Embajada y Consulados de Estados Unidos en México. Disponible en: https://mx.usembassy.gov/es/discurso-del-secretario-de-estado-rex-tillerson-en-la-conferencia-sobre-prosperidad-y-seguridad-en-america-central/

TILLERSON, R., KELLY, J. y MORENO, L. (2017). «Construir prosperidad garantiza seguridad en América Central». Embajada y Consulados de Estados Unidos en México. Disponible en: https://mx.usembassy.gov/es/construir-prosperidad-garantiza-seguridad-en-america-central/ (consultado, 19 de junio de 2017).

UNODC-GOBIERNO DE COLOMBIA (2017). «Colombia. Monitoreo de territorios afectados por cultivos ilícitos 2016». Recuperado de: https://www.unodc.org/documents/colombia/2017/ julio/CENSO_2O17_WEB_baja.pdf 